\title{
ON THE SOLUTION OF NEVANLINNA PICK PROBLEM WITH SELFADJOINT EXTENSIONS OF SYMMETRIC LINEAR RELATIONS IN HILBERT SPACE
}

\author{
A. A. EL-SABBAGH \\ Department of Mathematics \\ Faculty of Engineering \\ Zagazig University, Shoubra \\ 108 Shoubra Street, Cairo, Egypt
}

(Received June 19, 1995 and in revised form December 1, 1995)

\begin{abstract}
The representation of Nevanlinna Pick Problem is well known, see [7], [8] and [11]. The aim of this paper is to find the necessary and sufficient condition for the solution of Nevanlinna Pick Problem and to show that there is a one-to-one correspondence between the solutions of the Nevanlinna Pick Problem and the minimal selfadjoint extensions of symmetric linear relation in Hilbert space. Finally, we define the resolvent matrix which gives the solutions of the Nevanlinna Pick Problem.
\end{abstract}

KEY WORDS AND PHRASES: Hilbert space, operators, symmetric linear relations, compressed resolvent, selfadjont extensions and Nevanlinna pick problem.

1991 AMS SUBJECT CLASSIFICATION CODES: 47A20.

\section{INTRODUCTION}

The Nevanlinna Pick Problem consists of finding a function $f(\ell), \quad \ell \in \mathbb{C}$, analytic in $\operatorname{Im} \ell>0$, $\operatorname{Im}(\ell)$ having a fixed sign there, to take assigned values at an infinite sequence of points in $\operatorname{Im}(\ell)>0$, see [13].

We define the Nevanlinna class $I \mathrm{~N}^{n}$ as the class of all $n \times n$ matrix functions $N(\ell)$, which are holomorphic in $\mathbb{C} \backslash \mathbb{R}$, satisfying $N(\ell)^{*}=N(\bar{\ell}), \quad \ell \in \mathbb{C} \backslash \mathbb{R}$, and for which the kernel: $K_{N}(\ell, \lambda)=\frac{N(\ell)-N(\lambda)^{\bullet}}{\ell-\bar{\lambda}}, \ell, \lambda \in \mathbb{C} \backslash \mathbb{R} \ell \neq \bar{\lambda}$, is nonnegative.

It is well known that for each $N(\ell) \in \mathbb{N}^{n}$ there exist $n \times n$ matrices $A$ and $B$ with $A=A^{*}$ and $B=B^{*} \geqq 0$, and a nondecreasing $n \times n$ matrix function $\sum$ on $\mid \mathrm{R}$ with $\int_{\mathbb{R}}\left(t^{2}+1\right)^{-1} d \sum(t)<\infty$, such that: $N(\ell)=A+B \ell+\int_{\mathbb{R}}\left(\frac{1}{t-\ell}-\frac{t}{t^{2}+1}\right) d \sum(t), \quad \ell \in \mathbb{C} \backslash \mathbb{R}$.

With this so-called Riesz-Herglotz representation the kernel $K_{N}(\ell, \lambda)$ takes the form $K_{N}(\ell, \lambda)=$ $B+\int_{\mathbb{R}} \frac{d \sum(t)}{(t-\ell)(t-\bar{\lambda})}, \quad \ell, \lambda \in \mathbb{C} \backslash \mathbb{R}$.

This is the general case, but now our representation for Nevanlinna function takes such different cases.

To construct the solution function of the Nevanlinna Pick Problem in this paper we define the suitable Hilbert space which we need and define on it the linear relation which is symmetric and find the minimal selfadjoint extension of that relation in order to show that there is a one-to-one correspondence between the solutions of the Nevanlinna Pick Problem and the minimal selfadjoint 
extensions of the symmetric linear relation we have. This will form the contents of the first two sections. In the last section, we give a description of the resolvent matrix which gives the solutions of the Nevanlinna Pick Problem and shows that there is a one-to-one correspondence between the solutions of the Nevanlinna Pick Problem and all Nevanlinna pairs $(P L)$ defined in [8], [9] and [10].

In this paper we shall use the following notations:

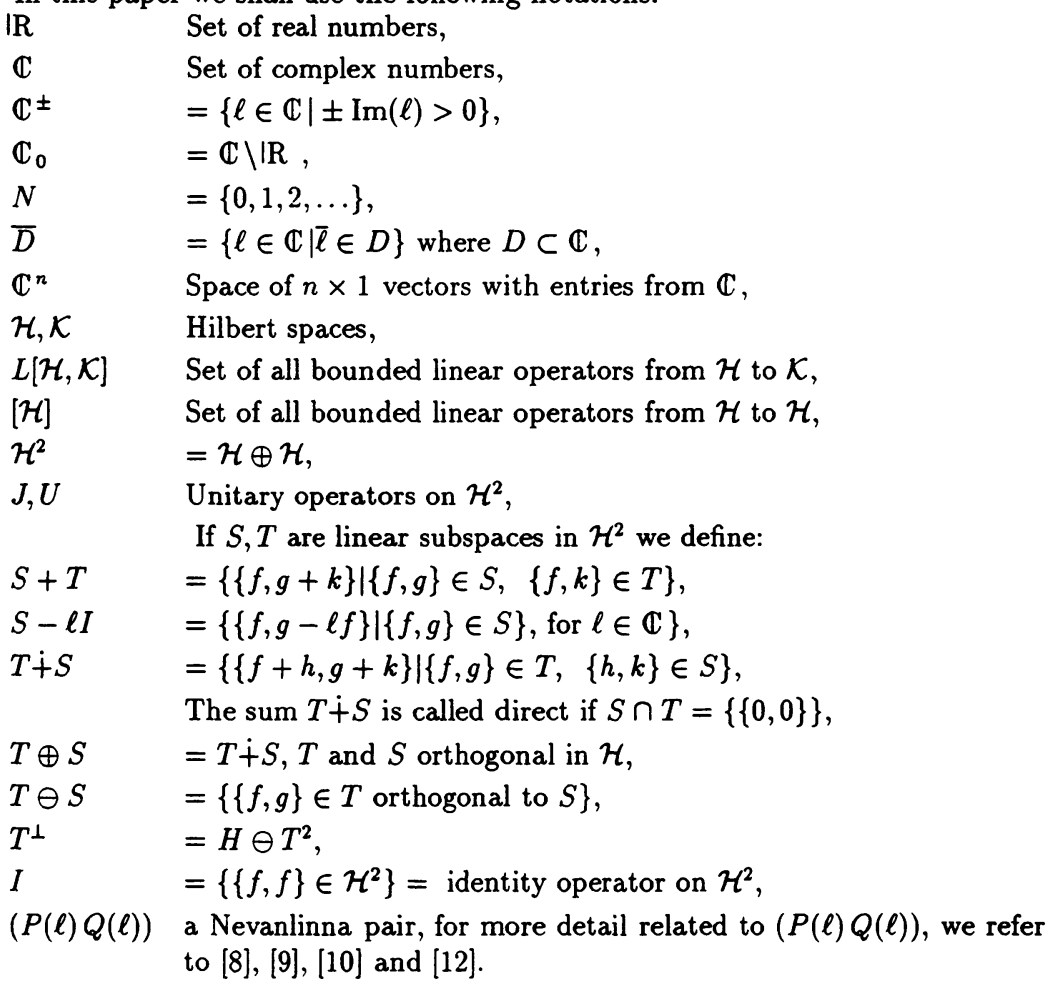

\section{PRELIMINARIES}

In this section, we collect several basic observations concerning our subject. If $\mathcal{H}$ is a Hilbert space over the complex numbers $\mathbb{C}$, we let $\mathcal{H}^{2}=\mathcal{H} \oplus \mathcal{H}$, considered as the Hilbert space of all pairs $\{f, g\}$ with $f, g \in \mathcal{H}$. A linear relation in $\mathcal{H}$ is a linear manifold $T$ in $\mathcal{H}^{2}$. The domain of $T, D(T)$, is defined by $D(T)=\{f \in \mathcal{H} \mid\{f, g\} \in T$ for some $g \in \mathcal{H}\}$, and the range of $T, R(T)$, is given by $R(T)=\{g \in \mathcal{H} \mid\{f, g\} \in T$ for some $f \in \mathcal{H}\}$. For $f \in R(T)$, we let $T(f)=\{g \mid\{f, g\} \in T\}$, and thus $R(T)$ is the union of all $T(f)$, for $f \in R(T)$. The inverse of $T, T^{-1}$, is the linear relation $T^{-1}=\{\{g, f\} \mid\{f, g\} \in T\}$. The null space of $T, \nu(T)$, is defined by $\nu(T)=\{f \in \mathcal{H} \mid\{f, 0\} \in T\}$. A linear relation $T$ in $\mathcal{H}$ is a (linear) operator in $\mathcal{H}$ if $T(0)=\{0\}$. If $T$ is an operator in $\mathcal{H}$ we shall abbreviate $T(f)$ by the usual $T f$.

If $S$ and $T$ are linear relations in $\mathcal{H}$, we define their product $S T$ by $S T=\{\{f, k\} \mid\{f, g\} \in$ $T,\{g, k\} \in S$, for some $g \in \mathcal{H}\}$. For each $\alpha \in \mathbb{C}$ we can associate an operator in $\mathcal{H}$ (which can be thought of as $\alpha$ times the identity operator $I$ ) given by $\{\{f, \alpha f\} \mid f \in \mathcal{H}\}$. If we identify this operator with $\alpha$, then we have $\alpha T=\{\{f, \alpha g\} \mid\{f, g\} \in T\}$.

The linear relations which are of most interest to us are those which are closed linear manifolds in $\mathcal{H}^{2}$, and we shall call these just subspaces in $\mathcal{H}^{2}$. Unless otherwise stated, all of the linear relations we consider in the remainder of this work will be subspaces. 
The adjoint $T^{*}$ of a subspace $T$ in $\mathcal{H}^{2}$ is defined as the linear relation

$$
T^{*}=\left\{\{h, k\} \in \mathcal{H}^{2} \mid[g, h]=[f, k], \text { for all }\{f, g\} \in T\right\} .
$$

Here $[\cdot, \cdot]$ denotes the inner product defined in $\mathcal{H}$. A convenient way to analyze the properties of the adjoint is to introduce the two operators $J, U$ defined on all of $\mathcal{H}^{2}$ as follows:

$$
\begin{aligned}
& J\{f, g\}=\{g,-f\}, \\
& U\{f, g\}=\{g, f\} .
\end{aligned}
$$

Both $J$ and $U$ are unitary operators on $\mathcal{H}^{2}$, and it is easily checked that

$$
\begin{aligned}
& J^{2}=-I, U^{2}=I, U J=-J U, \\
& T^{*}=\mathcal{H}^{2} \ominus J T=(J T)^{\perp}=J\left(T^{\perp}\right), \\
& T^{-1}=U T .
\end{aligned}
$$

We refer to [2], [3], [4] and [5].

The subspace $T \subset \mathcal{H}^{2}$ is called symmetric if $T \subset T^{*}$ and selfadjoint if $T=T^{*}$. Recall that if $T \subset \mathcal{H}^{2}$ is a subspace, the resolvent set $\rho(T)$ of $T$ is defined by

$$
\rho(T)=\left\{\ell \in \mathbb{C} \mid(T-\ell)^{-1} \in[\mathcal{H}]\right\},
$$

and the resolvent operator $R_{T}(\ell): \rho(T) \rightarrow[\mathcal{H}]$ of $A$ is defined by

$$
R_{T}(\ell)=(T-\ell)^{-1}, \quad \ell \in \rho(T)
$$

A symmetric subspace $S$ in $\mathcal{H}^{2}$ always has selfadjoint extensions in a suitably larger Hilbert space, but there exist selfadjoint extensions of $S$ in $\mathcal{H}^{2}$ if and only if for some $\ell \in \mathbb{C}^{+}$(and hence for all $\ell \in \mathbb{C}^{+}$) the defect numbers of $S$ are equal. Let $\mathcal{A}$ be selfadjoint extension of $S$ in a larger Hilbert space $\mathcal{K}, \mathcal{K} \supset \mathcal{H}$-with nonempty resolvent set $\rho(\mathcal{A})$. Then on $\rho(\mathcal{A})$ we study the locally holomorphic $[\mathcal{H}]$-valued function $R_{1}$ defined by

$$
\begin{aligned}
R_{1}(\ell) & =\left.P(\mathcal{A}-\ell)^{-1}\right|_{\mathcal{H}} \\
& =\{\{g-\ell f, P f\} \mid\{f, g\} \in \mathcal{A}, g-\ell f \in \mathcal{H}\}, \quad \ell \in \rho(\mathcal{A}),
\end{aligned}
$$

where $P$ denotes the orthogonal projection from $\mathcal{K}$ onto $\mathcal{H}(\mathcal{K} \supset \mathcal{H})$. This function $R_{1}$ is called the compressed resolvent of $\mathcal{A}$ in $\mathcal{H}$. If $S$ is symmetric, one can easily verify that $R_{1}(\ell)$ is a holomorphic mapping with values in $[\mathcal{H}]$, with domain of holomorphy $D_{R_{1}}$ which is symmetric with respect to the real axis: $D_{R_{1}}=D_{R_{1}}^{*}$, also $R_{1}(\ell)^{*}=R_{1}(\bar{\ell})$. Finally $\left\{R_{1}(\ell) f, \ell R_{1}(\ell) f+f\right\} \in S^{*}$ for all $f \in \mathcal{H}$, see [6]. In this case the compressed resolvent $R_{1}(\ell)$ of $\mathcal{A}$ in $\mathcal{H}$ is called a generalized resolvent of $S$, or the generalized resolvent of $S$ associated with $\mathcal{A}$, see [6] and [7].

Finally, if $T \supset \mathcal{H}^{2}$ is a closed linear relation, $\nu \in \mathbb{C}_{0}$. We define the Cayley transform $C_{\nu}(T)$ of $T$ and the inverse Cayley transform $F_{\nu}(T)$ of $T$ with respect to $\nu$ by:

$$
\begin{aligned}
& C_{\nu}(T)=\{\{g-\nu f, g-\bar{\nu} f\} \mid\{f, g\} \in T\} \\
& F_{\nu}(T)=\{\{g-f, \nu g-\bar{\nu} f\} \mid\{f, g\} \in T\} .
\end{aligned}
$$

We refer to [1], [4] and [14]. We may see that the description of Coddington for all selfadjoint extensions in possibly larger spaces is based on the corresponding results for unitary extensions of isometric operators, see [6]. 


\section{SOLUTION OF THE NEVANLINNA PICK PROBLEM}

Our interest will be in selfadjoint extensions of a given symmetric subspace (closed linear relation) and this has been discussed, for instance see [3], [5], and [6], when one of that extensions is minimal and its connection with Resolvent has been discussed in [9], [10, [12] and [13]. Then taking into consideration [5], [6] and [7], we come to the following.

THEOREM 3.1. If we have two sets of points $Z_{0}$ and $W_{0}$ defined as: $Z_{0}=\left\{z_{i} \mid z_{i} \in \mathbb{C}^{+}, i=\right.$ $1,2, \ldots, n\}, W_{0}=\left\{\omega_{i} \mid \omega_{\imath} \in \mathbb{C}^{+}, i=1,2, \ldots, n\right\}$ and the matrix $G_{\imath \jmath}$ defined as $G_{i j}=\frac{\omega_{i}-\bar{\omega}_{j}}{z-\bar{z}_{j}}$, if the matrix $G_{i j} \geqq 0$, we can find an $f$ defined from $\mathbb{C}^{+}$up $\mathbb{C}^{+}$such that $f\left(z_{i}\right)=\omega_{i}$, that will solve the Nevanlinna pick problem.

PROOF.

1. Necessity: Since $f \in \mathbb{N}^{n}$, then $f$ permits the representation

$$
\begin{aligned}
f(z)= & \mu z+\nu+\int_{\mathbb{R}} \frac{1+t z}{t-z} d \sigma(t) \quad \text { (see [1]) } \\
& \text { with } \quad \mu, \nu \in \mathbb{R}, \mu \geq 0, \quad \sigma(t) \quad \text { nondecreasing. } \int \sigma(t)<\infty . \\
\Longrightarrow & {\left[\frac{\omega_{i}-\bar{\omega}_{\jmath}}{z_{\imath}-\bar{z}_{\jmath}}\right]=\mu+\int_{\mid \mathrm{R}} \frac{1+t^{2}}{\left(t-z_{\imath}\right)\left(t-\bar{z}_{\jmath}\right)} d \sigma(t) } \\
\Longrightarrow & \sum_{i, j} G_{i j} x, \bar{x}_{\imath}=\mu\left|\sum_{i} x_{\imath}\right|^{2}+\int_{\mathbb{R}}\left|\sum \frac{x_{\imath}}{t-z_{\imath}}\right|^{2}\left(t^{2}+1\right) d \sigma(t) \geq 0 .
\end{aligned}
$$

2. Sufficient. We build the Hilbert space $\mathcal{H}$ as

$$
\mathcal{H}=\left\{\sum x_{i} \epsilon_{\imath} \mid x_{\imath} \in \mathbb{C}, \quad i=1, \ldots, n\right\}
$$

and two inner products on $\mathcal{H}$ defined by

$$
(y, x)=\sum \bar{x}_{\imath} y_{\imath}, \quad x, y \in \mathbb{C}^{n} .
$$

So

$$
\begin{gathered}
{[y, x]=(G y, x)=\sum \bar{x}_{\imath} G_{\imath \jmath} y_{\jmath}} \\
{\left[\epsilon_{\imath}, \epsilon_{\jmath}\right]=G_{\jmath \imath}=\frac{\omega_{\jmath}-\bar{\omega}_{\imath}}{z_{\jmath}-\bar{z}_{\imath}} .}
\end{gathered}
$$

Define the linear operators $Z$ and $W$ on $\mathcal{H}$ and a relation $S$ in $\mathcal{H}^{2}$ by

$$
\begin{aligned}
Z\left[\epsilon_{\imath}\right] & =\bar{z}_{\imath} e_{i} \\
W\left[\epsilon_{\imath}\right] & =\bar{\omega}_{\imath} \epsilon_{\imath} \\
S & =\left\{\{x, Z x\} \mid x=\sum_{\imath} x_{\imath} \epsilon_{\imath}, \sum_{u} x_{\imath}=0\right\} \\
e & =(1,1,1, \ldots, 1)=\sum \epsilon_{\imath} \\
u & =(1,0,0, \ldots, 0)=\epsilon_{1} .
\end{aligned}
$$

Straightforward calculations will show that $S$ is symmetric.

$$
\begin{aligned}
{[Z y, x]-[y, Z x] } & =\sum \bar{x}_{i} G_{\imath}, \bar{z}_{\jmath} y_{\jmath}-\sum \bar{x}_{i} z_{\imath} G_{i \jmath} y_{\jmath} \\
& =\sum \bar{x}_{i} y_{\jmath} G_{\imath \jmath}\left(\bar{z}_{\jmath}-z_{\jmath}\right) \\
& =\sum \bar{x}_{\imath} y_{\jmath}\left(\bar{w}_{\jmath}-\omega_{\imath}\right) \\
& =\sum \bar{x}_{\imath} y_{\jmath} \bar{\omega}_{\jmath}-\sum \bar{x}_{i} y_{\jmath} \omega_{\imath} \\
& =\left(\sum \bar{x}_{\imath}\right)\left(\sum y, \bar{\omega}_{\jmath}\right)-\left(\sum \bar{x}_{\imath} \omega_{\imath}\right)\left(\sum y_{\imath}\right) \\
& =(e, x)(W y, e)-(e, W x)(y, e) .
\end{aligned}
$$


This implies that for $x$ and $y \in D(S)$ that is $(x, e)=(y, e)=0$, that

$$
[Z y, x]-[y, Z x]=0 .
$$

Let $A$ be a minimal selfadjoint extension of $S$. With this $A$ we will now define a solution $f(z)$ of the original interpolation problem and show that this $f(z)$ satisfies all the conditions.

$$
f(\ell)=\omega_{1}+\left(\ell-z_{1}\right) G_{11}+\left(\ell-z_{1}\right)\left(\ell-\bar{z}_{1}\right)\left[(A-\ell)^{-1} u, u\right]
$$

1. $f\left(z_{1}\right)=\omega_{1}$ trivial

2. $f\left(z_{j}\right)=\omega_{j}$

$$
f\left(z_{\jmath}\right)=\omega_{1}+\left(z_{\jmath}-z_{1}\right) G_{11}+\left(z_{\jmath}-z_{1}\right)\left(z_{\jmath}-\bar{z}_{1}\right)\left[u,\left(A-\bar{z}_{\jmath}\right)^{-1} u\right]
$$

We know: $\left(Z-\bar{z}_{\jmath}\right)\left(\epsilon_{1}-\epsilon_{\jmath}\right)=\left(\bar{z}_{1}-\bar{z}_{\jmath}\right) \epsilon_{1}+\left(\bar{z}_{\jmath}-\bar{z}_{\jmath}\right) \epsilon_{\jmath}$

$$
\begin{aligned}
& \Longrightarrow\left(S-\bar{z}_{\jmath}\right)\left(\epsilon_{1}-\epsilon_{\jmath}\right)=\left(\bar{z}_{1}-\bar{z}_{\jmath}\right) \epsilon_{i} \\
& \Longrightarrow\left(A-\bar{z}_{\jmath}\right)^{-1} \epsilon_{1}=\left(\epsilon_{1}-\epsilon_{\jmath}\right)\left(\bar{z}_{1}-\bar{z}_{\jmath}\right)^{-1} \\
f\left(z_{\jmath}\right) & =\omega_{1}+\left(z_{\jmath}-z_{1}\right) G_{11}+\left(z_{\jmath}-z_{1}\right)\left(z_{\jmath}-\bar{z}_{1}\right)\left[\epsilon_{1},\left(\epsilon_{1}-\epsilon_{\jmath}\right)\left(\bar{z}_{1}-\bar{z}_{\jmath}\right)^{-1}\right] \\
& =\omega_{1}+\left(z_{\jmath}-z_{1}\right) G_{11}-\left(z_{\jmath}-\bar{z}_{1}\right)\left[\epsilon_{1},\left(\epsilon_{1}-\epsilon_{\jmath}\right)\right] \\
& =\omega_{1}+\left[\left(z_{j}-z_{1}\right)\left(z_{\jmath}-\bar{z}_{1}\right)\right] G_{11}+\left(z_{\jmath}-\bar{z}_{1}\right)\left[\epsilon_{1}, \epsilon_{\jmath}\right] \\
& =\omega_{1}+\bar{\omega}_{1}-\omega_{1}+\omega_{\jmath}-\bar{\omega}_{1} \\
& =\omega_{\jmath}
\end{aligned}
$$

3. $\operatorname{Im}(\ell)>0 \Rightarrow \operatorname{Im}(f(\ell))>0$

$$
\begin{aligned}
& \omega_{1}+\left(\ell-z_{1}\right) G_{11}-\bar{\omega}_{1}-\left(\bar{\ell}-\bar{z}_{1}\right) G_{11}=(\ell-\bar{\ell}) G_{11}=(\ell-\bar{\ell})[u, u] \\
& \quad\left(\ell-\bar{z}_{1}\right)\left(\ell-z_{1}\right)(A-\ell)^{-1} \\
&=\quad(A-\ell)^{-1}\left(\ell-A+A-z_{1}\right)\left(\ell-\bar{z}_{1}\right) \\
&=-\left(\ell-\bar{z}_{1}\right)+\left(A-z_{1}\right)(A-\ell)^{-1}\left(\ell-A+A-\bar{z}_{1}\right) \\
&=-\left(\ell-\bar{z}_{1}\right)-\left(A-z_{1}\right)+\left(A-z_{1}\right)(A-\ell)^{-1}\left(A-\bar{z}_{1}\right) \\
& \Rightarrow \quad\left(\ell-\bar{z}_{1}\right)\left(\ell-z_{1}\right)(A-\ell)^{-1}-\left(\bar{\ell}-z_{1}\right)\left(\bar{\ell}-\bar{z}_{1}\right)(A-\bar{\ell})^{-1} \\
&=-\ell+\bar{z}_{1}-A+z_{1}+\left(A-z_{1}\right)(A-\ell)^{-1}\left(A-\bar{z}_{1}\right)+\bar{\ell}-z_{1}+A-\bar{z}_{1}+ \\
& \quad+\left(A-\bar{z}_{1}\right)(A-\bar{\ell})^{-1}\left(A-z_{1}\right) \\
&= \bar{\ell}-\ell+\left(A-z_{1}\right)(A-\ell)^{-1}\{(A-\bar{\ell})-(A-\ell)\}(A-\bar{\ell})^{-1}\left(A-\bar{z}_{1}\right) \\
&=(\ell-\bar{\ell})\left[\left(A-z_{1}\right)(A-\ell)^{-1}(A-\bar{\ell})^{-1}\left(A-\bar{z}_{1}\right)-I\right] \\
& \Rightarrow \operatorname{Im}(f(\ell)) / \operatorname{Im}(\ell)=\left[\left(A-z_{1}\right)(A-\ell)^{-1}(A-\bar{\ell})^{-1}\left(A-\bar{z}_{1}\right) u, u\right] \\
& \quad=\left[(A-\bar{\ell})^{-1}\left(A-\bar{z}_{1}\right) u,(A-\bar{\ell})^{-1}\left(A-\bar{z}_{1}\right) u\right] \geq 0 .
\end{aligned}
$$

4. $f(\ell)$ is analytic for $\ell \in \mathbb{C}^{+}$.

This follows immediately from the fact that $A$ is selfadjoint. This completes the proof.

THEOREM 3.2. There exists a one-to-one correspondence between all solutions of the Nevanlinna Pick Problem constructed in THEOREM 3.1 and all minimal selfadjoint extensions of $S$.

PROOF. Assume $f(\ell)$ is a solution of the Nevanlinna Pick Problem

$\Rightarrow$ There exists a Hilbert space $(\mathcal{K},\langle\rangle$,$) , an element u$ and an unitary operator $U$ from $\mathcal{K}$ to $\mathcal{K}$ such that $f(\ell)=s+\left(z_{1}-\bar{z}_{1}\right)\left\langle(I+p(\ell) U)(I-p(\ell) U)^{-1} u, u\right\rangle$ with $s \in \mid \mathrm{R}$ and $p(\ell)=\left(\ell-z_{1}\right)\left(\ell-\bar{z}_{1}\right)^{-1}$, see [8]. 
We can take $\mathcal{K}$ minimal in which case $U$ is unique up to isomorphism

$$
f\left(z_{1}\right)=\omega_{1} \Rightarrow s=\operatorname{Re}\left(\omega_{1}\right) \text { and }\langle u, u\rangle=\left(\omega_{1}-\bar{\omega}_{1}\right)\left(z_{1}-\bar{z}_{1}\right)^{-1} .
$$

Take $A=F_{z_{1}}(U)$, the inverse Cayley transform of $U$.

$$
\Rightarrow f(\ell)=\omega_{1}+\left(\ell-z_{1}\right)\langle u, u\rangle+\left(\ell-z_{1}\right)\left(\ell-\bar{z}_{1}\right)\left\langle(A-\ell)^{-1} u, u\right\rangle
$$

We wish to show that $\exists \Gamma$ isomorphism $\mathcal{H} \rightarrow \mathcal{K}$ such that $\Gamma S \subseteq A \Gamma$.

$$
\begin{aligned}
\text { Define } \quad e_{1} & =u \\
e_{\jmath} & =e_{1}+\left(\bar{z}_{\jmath}-\bar{z}_{1}\right)\left(A-\bar{z}_{\jmath}\right)^{-1} e_{1} \quad j \geq 2 \\
& \Rightarrow\left(A-\bar{z}_{\jmath}\right)^{-1} e_{1}=\left(e_{\jmath}-e_{1}\right)\left(\bar{z}_{\jmath}-\bar{z}_{1}\right)^{-1} \\
& \Rightarrow A\left(e_{\jmath}-e_{1}\right)=\bar{z}_{\jmath} e_{\jmath}-\bar{z}_{1} e_{1} .
\end{aligned}
$$

$$
\text { Define } \quad \begin{aligned}
\Gamma \epsilon_{1}=e_{\imath} \text { and } A \Gamma\left(\epsilon_{\jmath}-\epsilon_{1}\right) & =A\left(e_{j}-e_{1}\right)=\bar{z}, \epsilon_{j}-\bar{z}_{1} e_{1} \\
& =\Gamma\left(\bar{z}_{\jmath} \epsilon_{\jmath}-\bar{z}_{1} \epsilon_{1}\right) \\
& =\Gamma S\left(\epsilon_{\jmath}-\epsilon_{1}\right) \quad \text { for } j=2, \ldots n \\
& \Rightarrow \Gamma S \subseteq A \Gamma
\end{aligned}
$$

$\left[\epsilon_{i}, \epsilon_{\jmath}\right]=\left\langle e_{\imath}, e_{\jmath}\right\rangle$, for these cases we have :

(a) $\left\langle e_{1}, e_{1}\right\rangle=\left(\omega_{1}-\bar{\omega}_{1}\right)\left(z_{1}-\bar{z}\right)^{-1}=\left[\epsilon_{1}, \epsilon_{1}\right]$

(b) $\left\langle e_{1}, e_{\jmath}\right\rangle$ can be constructed as follows:

$$
\begin{aligned}
f\left(z_{\jmath}\right) & =\omega_{\jmath} \Rightarrow \\
\omega_{\jmath} & =\omega_{1}+\left(z_{\jmath}-z_{1}\right)\langle u, u\rangle+\left(z_{\jmath}-z_{1}\right)\left(z_{\jmath}-\bar{z}_{1}\right)\left\langle\left(A-z_{\jmath}\right)^{-1} u, u\right\rangle \\
& =\omega_{1}+\left(z_{\jmath}-z_{1}\right)\langle u, u\rangle+\left(z_{\jmath}-z_{1}\right)\left(z_{\jmath}-\bar{z}_{1}\right)\left\langle u,\left(A-z_{\jmath}\right)^{-1} u\right\rangle \\
& =\omega_{1}+\left(z_{\jmath}-z_{1}\right)\left\langle e_{1}, e_{1}\right\rangle+\left(z_{\jmath}-z_{1}\right)\left(z_{\jmath}-\bar{z}_{1}\right)\left\langle e_{1},\left(e_{\jmath}-e_{1}\right)\left(\bar{z}_{j}-\bar{z}_{1}\right)^{-1}\right\rangle \\
& =\omega_{1}+\left(z_{\jmath}-z_{1}\right)\left\langle e_{1}, e_{1}\right\rangle+\left(z_{\jmath}-\bar{z}_{1}\right)\left\langle e_{1},\left(e_{\jmath}-e_{1}\right)\right\rangle . \\
& =\omega_{1}+\left(\bar{z}_{1}-z_{1}\right)\left\langle e_{1}, e_{1}\right\rangle+\left(z_{\jmath}-\bar{z}_{1}\right)\left\langle e_{1}, e_{\jmath}\right\rangle, \quad \text { So now : } \\
& \Rightarrow\left\langle e_{1}, e_{\jmath}\right\rangle=\left(\omega_{\jmath}-\bar{\omega}_{1}\right)\left(z_{j}-\bar{z}_{\jmath}\right)^{-1}=\left[\epsilon_{1}, \epsilon_{\jmath}\right] .
\end{aligned}
$$

(c) $\left\langle e_{\imath}, e_{\jmath}\right\rangle$ can be constructed as follows:

$$
\begin{aligned}
\left\langle e_{i}, e_{\jmath}\right\rangle & =\left\langle\left(e_{\jmath}-e_{1}\right),\left(e_{j}-e_{1}\right)\right\rangle+\left\langle e_{1}, e_{\jmath}\right\rangle+\left\langle e_{\imath}, e_{1}\right\rangle-\left\langle e_{1}, e_{1}\right\rangle \\
\left\langle\left(\mathrm{e}_{\mathrm{i}}-\mathrm{e}_{1}\right),\left(\mathrm{e}_{\mathrm{j}}-\mathrm{e}_{1}\right)\right) & =\left(\bar{z}_{i}-\bar{z}_{1}\right)\left(z_{\jmath}-z_{1}\right)\left\langle\left(A-\bar{z}_{\imath}\right)^{-1} e_{1},\left(A-\bar{z}_{\jmath}\right)^{-1} e_{1}\right\rangle \\
& =\left(\bar{z}_{\imath}-\bar{z}_{1}\right)\left(z_{\jmath}-z_{1}\right)\left\langle\left(A-z_{\jmath}\right)^{-1}\left(A-\bar{z}_{\imath}\right)^{-1} e_{1}, e_{1}\right\rangle \\
& =\left(\bar{z}_{\imath}-\bar{z}_{1}\right)\left(z_{\jmath}-z_{1}\right)\left(\bar{z}_{\imath}-z_{\jmath}\right)^{-1}\left\langle\left(A-z_{\jmath}\right)^{-1}\left(\bar{z}_{\imath}-z_{\jmath}\right)\left(A-\bar{z}_{\imath}\right)^{-1} e_{1}, e_{1}\right\rangle \\
& =\left(\bar{z}_{\imath}-\bar{z}_{1}\right)\left(z_{\jmath}-z_{1}\right)\left(\bar{z}_{\imath}-z_{\jmath}\right)^{-1}\left\langle\left(A-z_{\jmath}\right)^{-1}\left\{\left(A-z_{\jmath}\right)-\left(A-\bar{z}_{\imath}\right)\right\}\left(A-\bar{z}_{i}\right)^{-1} e_{1}, e_{1}\right\rangle \\
& =\left(\bar{z}_{\imath}-\bar{z}_{1}\right)\left(z_{\jmath}-z_{1}\right)\left(\bar{z}_{\imath}-z_{\jmath}\right)^{-1}\left\{\left\langle\left(A-\bar{z}_{\imath}\right)^{-1} e_{1}, e_{1}\right\rangle-\left\langle\left(A-z_{\jmath}\right)^{-1} e_{1}, e_{1}\right\rangle\right\} \\
& =\left(z_{\jmath}-z_{1}\right)\left(\bar{z}_{\imath}-z_{\jmath}\right)^{-1}\left\langle e_{\imath}-e_{1}, e_{1}\right\rangle-\left(\bar{z}_{\imath}-\bar{z}_{1}\right)\left(\bar{z}_{\imath}-z_{\jmath}\right)^{-1}\left\langle e_{1}, e_{\jmath}-e_{1}\right\rangle
\end{aligned}
$$


From the above, we get

$$
\begin{aligned}
\left\langle e_{i}, e_{j}\right\rangle= & \left\langle\left(e_{i}-e_{1}\right),\left(e_{\jmath}-e_{1}\right)\right\rangle+\left\langle e_{1}, e_{\jmath}\right\rangle+\left\langle e_{i}, e_{1}\right\rangle-\left\langle e_{1}, e_{1}\right\rangle \\
= & \left\langle e_{1}, e_{\jmath}\right\rangle\left(1-\left(\bar{z}_{i}-\bar{z}_{1}\right)\left(\bar{z}_{i}-z_{j}\right)^{-1}\right)+\left\langle e_{i}, e_{1}\right\rangle\left(1+\left(z_{\jmath}-z_{1}\right)\left(\bar{z}_{i}-z_{j}\right)^{-1}\right)+ \\
& \quad+\left\langle e_{1}, e_{1}\right\rangle\left(\left(\bar{z}_{i}-\bar{z}_{1}\right)\left(\bar{z}_{i}-z_{\jmath}\right)^{-1}-\left(z_{\jmath}-z_{1}\right)\left(\bar{z}_{i}-z_{\jmath}\right)^{-1}-1\right) \\
= & \left(\bar{z}_{i}-z_{\jmath}\right)^{-1}\left(\left\langle e_{1}, e_{\jmath}\right\rangle\left(\bar{z}_{1}-z_{\jmath}\right)+\left\langle e_{i}, e_{1}\right\rangle\left(\bar{z}_{i}-z_{1}\right)+\left\langle e_{1}, e_{1}\right\rangle\left(z_{1}-\bar{z}_{1}\right)\right) \\
= & \left(\bar{z}_{i}-z_{\jmath}\right)^{-1}\left(\bar{\omega}_{1}-\omega_{\jmath}+\bar{\omega}_{i}-\omega_{1}+\omega_{1}-\bar{\omega}_{1}\right) \\
= & \left(\bar{z}_{i}-z_{\jmath}\right)^{-1}\left(\bar{\omega}_{i}-\omega_{\jmath}\right) \\
= & {\left[\epsilon_{i}, \epsilon_{\jmath}\right] . }
\end{aligned}
$$

From (a), (b) and (c) and the above, every solution can be written in the form $f(\ell)=\omega_{1}+(\ell-$ $\left.z_{1}\right) G_{11}+\left(\ell-z_{1}\right)\left(\ell-\bar{z}_{1}\right)\left[(A-\ell)^{-1} u, u\right]$ with $A$ a selfadjoint extension of $S$. As we have seen before every selfadjoint extension gives a solution of the Nevanlinna Pick Problem This completes the proof of the one-to-one correspondence of $f(\ell)$ and $A$.

LEMMA 3.1. Let $Y=\left(y_{i j}\right)$ and $W=\left(w_{i \jmath}\right)$ with $i, j=1,2, \ldots$ and $(P(\ell) Q(\ell))$ a Nevanlinna pair. If $f(\ell)$ is defined as $f(\ell)=\left\{y_{11}(\ell) P(\ell)+y_{12}(\ell) Q(\ell)\right\}\left\{y_{21}(\ell) P(\ell)+y_{22}(\ell) Q(\ell)\right\}^{-1}$ gives a oneto-one correspondence between all solutions of the Nevanlinna Pick Problem and all Nevanlinna pairs $(P(\ell) Q(\ell))$. We refer to [11], [13] and [14].

LEMMA 3.2. If $W(\ell)$ is defined as $W(\ell)=\left[(A-\ell)^{-1} u, u\right]$, then $[(A-\ell) u, u]=\left\{w_{11}(\ell) P(\ell)+\right.$ $\left.w_{12}(\ell) Q(\ell)\right\}\left\{w_{21}(\ell) P(\ell)+w_{22}(\ell) Q(\ell)\right\}^{-1}$ gives a one-to-one correspondence between the minimal selfadjoint extensions of $S$ and all the Nevanlinna pairs $(P(\ell) Q(\ell))$. This $W(\ell)$ is called the resolvent matrix of $S$, see [9], [10] and [11].

DEFINITION 3.1. If the function $f(z)$ is defined as $f(\ell)=\omega_{1}+\left(\ell-z_{1}\right) G_{11}+\left(\ell-z_{1}\right)\left(\ell-\bar{z}_{i}\right) W(\ell)$ where $W(\ell)$ is defined in Lemma 3.2, then we define the solution matrix of the Nevanlinna Pick Problem as:

$$
Y(\ell)=\left[\begin{array}{cc}
\left(\ell-z_{1}\right)(\ell-\bar{z}) & \omega_{1}+\left(\ell-z_{1}\right) G_{11} \\
0 & 1
\end{array}\right] W(\ell) .
$$

The connection between the solution matrix $Y(\ell)$ and the resolvent matrix will be the coming paper.

ACKNOWLEDGEMENT. I would like to thank P. Bruinsma, A. Dijksma and H. De Snoo for support and advice during my stay in Groningen University.

\section{REFERENCES}

[1] ACHIEZER, N.I. and GLAZMAN, I.M., "Theorie der linearen Operatoren im Hilbertraum," 8th ed., Akademie Verlag, Berlin, 1981.

[2] ARENS, R., "Operational calculus of linear relations," Pacific J. Math. 11(1961), 9-23.

[3] BENNEWITZ, C., "Symmetric Relations on a Hilbert Space," Lecture Notes in Math. 28, Springer-Verlag (1972), 212-218.

[4] BOGNAR, J., "Indefinite Inner Product Spaces," Springer-Verlag, Berlin, 1974.

[5] CODDINGTON, E.A. and GILBERT, R.C. "Generalized resolvents of ordinary differential operators," Trans. Amer. Math. Soc. 93(1959), 216-241.

[6] CODDINGTON, E.A., "Extension theory of formally normal and symmetric subspaces," Mem. Amer. Math. Soc., 134, 1973. 
[7] KREIN, M.G. and LANGER, H., "Defect subspaces and generalized resolvents of a Hermitian operator in the space $\Pi_{k}$," Functional Anal. Appl. 5(1972/72), 136-146, 217-228.

[8] KREIN, M.G. and LANGER, H., Über die verallgemeinerten Resolventen und die charakterische Funktion eines isometrischen Operators im Raume $\Pi_{k}$. Colloquia mathematica societatis Janos Bolyai 5: Hilbert space operators and operator algebras (Tihany 1970), North Holland Publishing Company, Amsterdam-London 1972, 353-399.

[9] LANGER, H. and TEXTORIUS, B., "On generalized resolvents and $Q$-functions of symmetric linear relations (subspaces) in Hilbert space," Pacific J. Math. 72(1977), 135-165.

[10] LANGER, H. and B. TEXTORIUS, B., " $L$-resolvent matrices of symmetric linear relations with equal defect numbers: applications to canonical differential relations," Integral Equations and Operator Theory 5(1982), 208-243.

[11] SHONKWHITER, R., "On generalized resolvents and an integral representation of Nevanlinna," J. Math. Anal. App. 40(1972), 723-734.

[12] ŠTRAUS, A.V., "Generalized Resolvents of Symmetric Operators," Izvest Akad. Nauk SSSR Ser. Mat. 18(1954), 51-86.

[13] SERGE LANG WILLIAM CHERRY, "Topics in Nevanlinna Theory," Lecture Notes in Mathematics, 1433, Springer-Verlag.

[14] TAYLOR, A.E., "Introduction to Functional Analysis," Wiley, New York, 1958. 


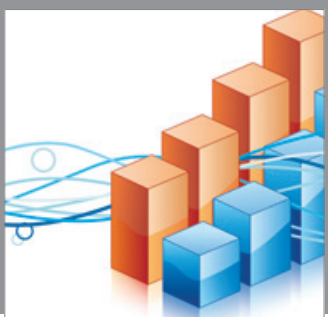

Advances in

Operations Research

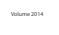

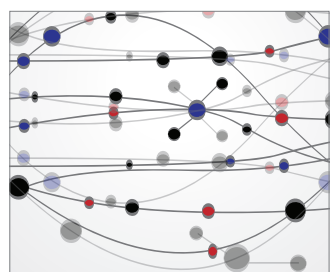

\section{The Scientific} World Journal
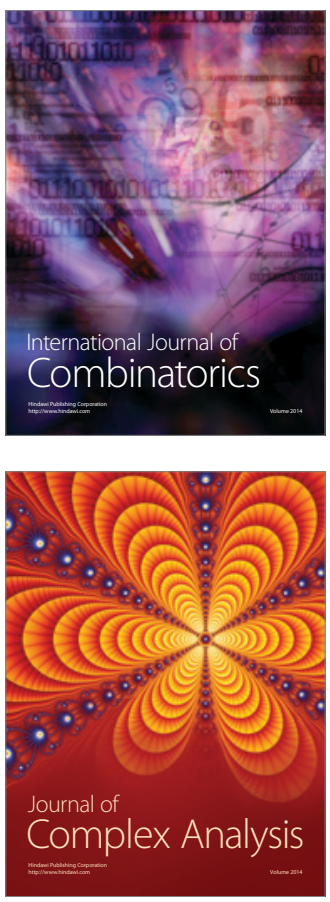

International Journal of

Mathematics and

Mathematical

Sciences
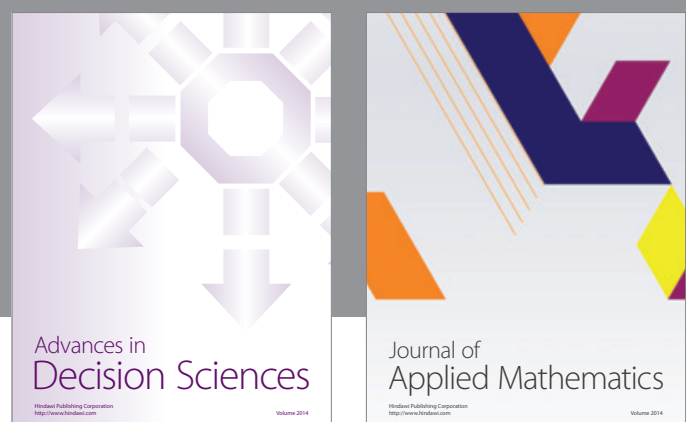

Journal of

Applied Mathematics
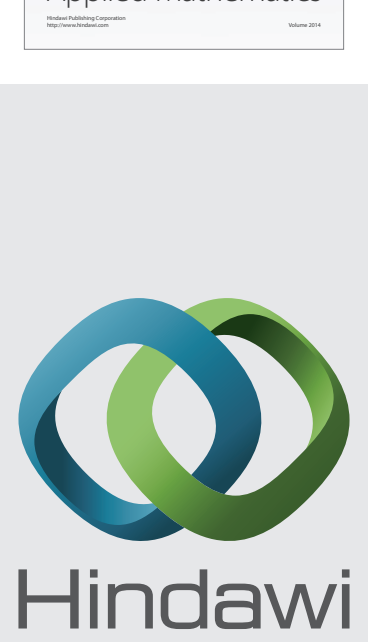

Submit your manuscripts at http://www.hindawi.com
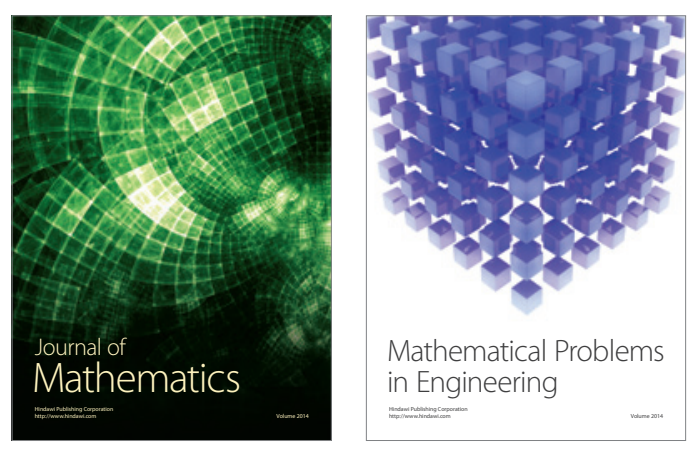

Mathematical Problems in Engineering
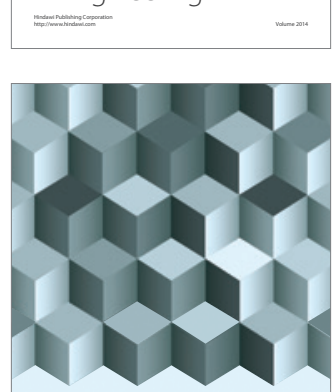

Journal of

Function Spaces
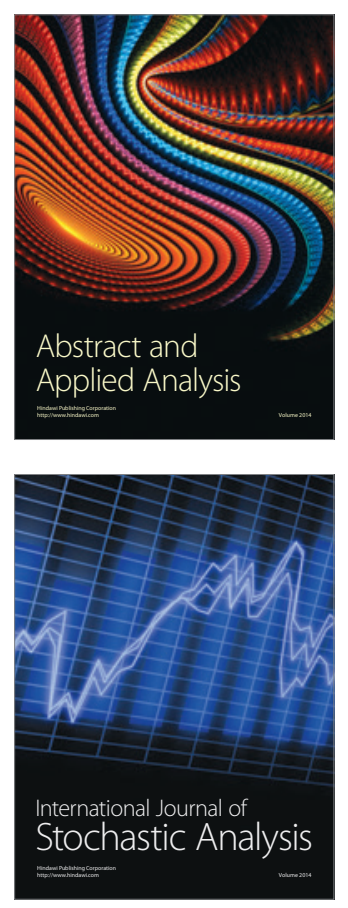

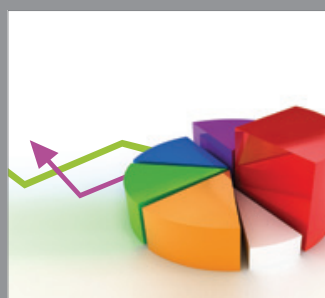

ournal of

Probability and Statistics

Promensencen
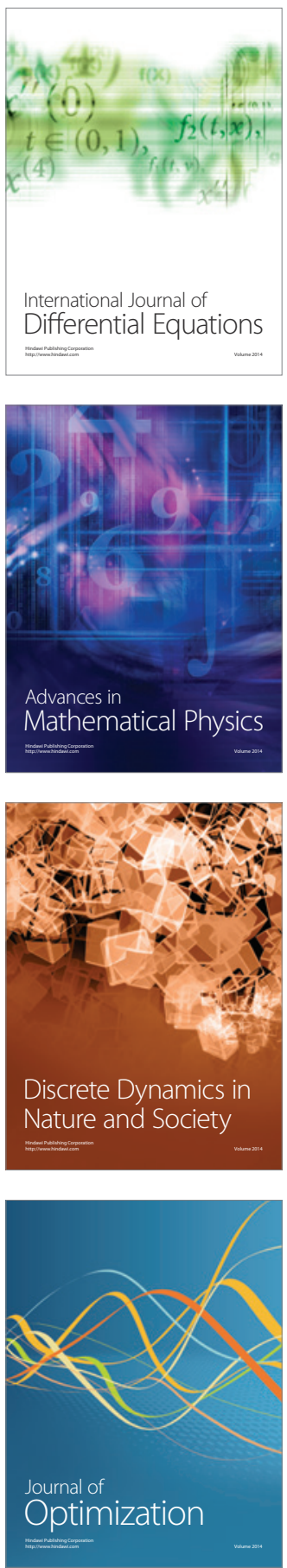